\title{
Diagnosis and outcome of acute respiratory failure in immunocompromised patients after bronchoscopy
}

Philippe R. Bauer ${ }^{1}$, Sylvie Chevret ${ }^{2}$, Hemang Yadav $^{1}$, Sangeeta Mehta ${ }^{3}$, Peter Pickkers ${ }^{4}$, Ramin B. Bukan ${ }^{5}$, Jordi Rello ${ }^{6}$, Andry van de Louw ${ }^{7}$, Kada Klouche ${ }^{8}$, Anne-Pascale Meert ${ }^{9}$, Ignacio Martin-Loeches ${ }^{10,11}$, Brian Marsh ${ }^{12}$, Lorenzo Socias Crespi $^{13}$, Gabriel Moreno-Gonzalez ${ }^{14}$, Nina Buchtele ${ }^{15}$, Karin Amrein ${ }^{16}$, Martin Balik ${ }^{17}$, Massimo Antonelli ${ }^{18}$, Martine Nyunga ${ }^{19}$, Andreas Barratt-Due ${ }^{20}$, Dennis C.J.J. Bergmans ${ }^{21}$, Angélique M.E. Spoelstra-de Man ${ }^{22}$, Anne Kuitunen ${ }^{23}$, Florent Wallet ${ }^{24}$, Amelie Seguin ${ }^{25}$, Victoria Metaxa ${ }^{26}$, Virginie Lemiale ${ }^{27}$, Gaston Burghi ${ }^{28}$, Alexandre Demoule ${ }^{29}$, Thomas Karvunidis ${ }^{30}$, Antonella Cotoia ${ }^{31}$, Pål Klepstad ${ }^{32}$, Ann M. Møller ${ }^{33}$, Djamel Mokart ${ }^{34}$ and Elie Azoulay ${ }^{27}$ for the Efraim investigators and the Nine-I study group ${ }^{35}$

@ERSpublications

In a pre-planned analysis of immunocompromised critically ill patients with acute respiratory failure, bronchoscopy was associated with better diagnosis and management but worse outcome. The decision to perform bronchoscopy should be individualised. http://bit.ly/2Dusahh

Cite this article as: Bauer PR, Chevret S, Yadav H, et al. Diagnosis and outcome of acute respiratory failure in immunocompromised patients after bronchoscopy. Eur Respir J 2019; 54: 1802442 [https://doi.org/ 10.1183/13993003.02442-2018].

\section{ABSTRACT}

Objective: We wished to explore the use, diagnostic capability and outcomes of bronchoscopy added to noninvasive testing in immunocompromised patients. In this setting, an inability to identify the cause of acute hypoxaemic respiratory failure is associated with worse outcome. Every effort should be made to obtain a diagnosis, either with noninvasive testing alone or combined with bronchoscopy. However, our understanding of the risks and benefits of bronchoscopy remains uncertain.

Patients and methods: This was a pre-planned secondary analysis of Efraim, a prospective, multinational, observational study of 1611 immunocompromised patients with acute respiratory failure admitted to the intensive care unit (ICU). We compared patients with noninvasive testing only to those who had also received bronchoscopy by bivariate analysis and after propensity score matching.

Results: Bronchoscopy was performed in 618 (39\%) patients who were more likely to have haematological malignancy and a higher severity of illness score. Bronchoscopy alone achieved a diagnosis in 165 patients (27\% adjusted diagnostic yield). Bronchoscopy resulted in a management change in 236 patients $(38 \%$ therapeutic yield). Bronchoscopy was associated with worsening of respiratory status in 69 (11\%) patients. Bronchoscopy was associated with higher ICU ( $40 \%$ versus $28 \%$; $<0.0001)$ and hospital mortality $(49 \%$ versus $41 \% ; \mathrm{p}=0.003$ ). The overall rate of undiagnosed causes was $13 \%$. After propensity score matching, bronchoscopy remained associated with increased risk of hospital mortality (OR 1.41, 95\% CI 1.08-1.81).

Conclusions: Bronchoscopy was associated with improved diagnosis and changes in management, but also increased hospital mortality. Balancing risk and benefit in individualised cases should be investigated further. 


\section{Introduction}

In immunocompromised patients, acute hypoxaemic respiratory failure is a frequent complication leading to $\sim 50 \% 90$-day mortality [1-4]. Inability to identify the cause of respiratory failure is associated with worse outcome [5]. Therefore, every effort should be made to obtain a diagnosis by noninvasive and, if necessary, invasive testing such as bronchoscopy [6]. In patients with malignancy, $90 \%$ of acute respiratory distress syndrome is due to infections [7]. Due to the poor tolerance to hypoxaemia, the effectiveness of bronchoscopy may be mitigated by the risk of respiratory deterioration and the need for ventilator support [5,8]. Bronchoscopy is certainly useful in some instances, such as when a nasopharyngeal viral panel is falsely negative [9] or when induced sputum to assess, for example, Pneumocystis jirovecii cannot be obtained. Overall, the rate of intubation for respiratory failure can reach $35 \%$ after bronchoscopy [5] and the rate of unidentified aetiologies remains substantial [10].

Most studies regarding bronchoscopy have been performed in relatively small cohorts of patients and may have been underpowered to detect any benefit or harm associated with the bronchoscopy itself $[5,8,10$, 11]. Moreover, the yield of bronchoscopy declines rapidly with time after clinical presentation [12]. Early use of broad-spectrum antimicrobials may reduce the likelihood of a positive microbiological diagnosis. Thus, the added utility of early bronchoscopy to noninvasive testing alone is still uncertain. On the one hand, clinicians may be reluctant to perform bronchoscopy in hypoxaemic patients because of concern for respiratory deterioration. On the other hand, they may feel compelled to perform bronchoscopy in spite of a low pre-test probability for fear of missing an unrecognised pathogen or aetiology (e.g. alveolar haemorrhage). Therefore, a contemporary study on a larger cohort of patients offered a unique opportunity to explore the use, diagnostic capability and outcomes of bronchoscopy when added to noninvasive testing.

We performed a pre-planned secondary analysis of the Efraim study, a prospective, multinational, observational study on acute hypoxaemic respiratory failure in immunocompromised patients, performed by the Nine-I (CarIng for CrItIcally Ill Immuno-compromIsed PatIents MultInatIonal Network) investigators [13]. Our objective was to address the diagnostic utility of bronchoscopy when added to noninvasive testing, the influence of bronchoscopy results on patient management, the association between

Affiliations: ${ }^{1}$ Division of Pulmonary and Critical Care Medicine, Mayo Clinic, Rochester, MN, USA. ${ }^{2}$ ECSTRA Team, Biostatistics and Clinical Epidemiology, UMR 1153, INSERM, Paris Diderot Sorbonne University and Service de Biostatistique et Information Médicale, AP-HP, Hôpital Saint-Louis, Paris, France. ${ }^{3}$ Dept of Medicine and Interdepartmental Division of Critical Care Medicine, Sinai Health System, University of Toronto, Toronto, ON, Canada. ${ }^{4}$ Dept of Intensive Care Medicine, Radboud University Medical Center, Nijmegen, The Netherlands. ${ }^{5}$ Dept of Anesthesiology I, Herlev University Hospital, Herlev, Denmark. ${ }^{6}$ CIBERES, Instituto Salud Carlos III and Vall d'Hebron Institut of Research Barcelona, Barcelona, Spain. ${ }^{7}$ Division of Pulmonary and Critical Care, Penn State University College of Medicine, Hershey, PA, USA. ${ }^{8}$ Dept of Intensive Care Medicine, Lapeyronie University Hospital, Montpellier, France. ${ }^{9}$ Service de Médecine Interne, Unité de Soins Intensifs et Urgences Oncologiques, Université de Libre de Bruxelles, Institut Jules Bordet, Brussels, Belgium. ${ }^{10}$ Dept of Intensive Care Medicine, Universidad de Barcelona IDIBAPS, Barcelona, Spain. ${ }^{11}$ Dept of Clinical Medicine, Trinity College, Wellcome Trust-HRB Clinical Research Facility, St James Hospital, Dublin, Ireland. ${ }^{12}$ Dept of Critical Care, Mater Misericordiae, Dublin, Ireland. ${ }^{13}$ Dept of Critical Care, Hospital Son Llatzer, Palma de Mallorca, Spain. ${ }^{14}$ Dept of Intensive Care, Hospital Universitari de Bellvitge, Barcelona, Spain. ${ }^{15}$ Dept of Clinical Pharmacology, Medical University of Vienna, Vienna, Austria. ${ }^{16}$ Dept of Internal Medicine, Division of Endocrinology and Diabetology, Medical University of Graz and Thyroid Endocrinology Osteoporosis Institute Dobnig, Graz, Austria. ${ }^{17}$ Dept of Anesthesiology and Intensive Care Medicine, First Faculty of Medicine, Charles University and General University Hospital, Prague, Czech Republic. ${ }^{18}$ Dept of Anesthesiology Intensive Care and Emergency Medicine, Fondazione Policlinico Universitario A. Gemelli IRCCS-Università Cattolica del Sacro Cuore, Rome, Italy. ${ }^{19}$ Medical Intensive Care Unit, CHG Victor Provo, Roubaix, France. ${ }^{20}$ Dept of Emergencies and Critical Care, Oslo University Hospital, Rikshospitalet, Oslo, Norway. ${ }^{21}$ Dept of Intensive Care, Maastricht University Medical Centre, Maastricht, The Netherlands. ${ }^{22}$ Dept of Intensive Care, Amsterdam UMC, VU Medical Center, Amsterdam. The Netherlands. ${ }^{23}$ Dept of Intensive Care, Tampere University Hospital, Tampere, Finland. ${ }^{24}$ Dept of Critical Care, University Hospital Lyon Sud, Pierre Benite, France. ${ }^{25}$ Réanimation Médicale, CHU de Caen, Caen, France. ${ }^{26}$ Dept of Critical Care, King's College Hospital NHS Foundation Trust, London, UK. ${ }^{27}$ Medical Intensive Care Unit, AP-HP, Hôpital SaintLouis, Famirea Study Group, ECSTRA Team, and Clinical Epidemiology, UMR 1153, Center of Epidemiology and Biostatistics, Sorbonne Paris Cité, CRESS, INSERM, Paris Diderot Sorbonne University, Paris, France.

${ }^{28}$ Terapia Intensiva, Hospital Maciel, Montevideo, Uruguay. ${ }^{29}$ Service de Pneumologie et Réanimation, CHU Pitié-Salpétrière, Paris, France. ${ }^{30}$ Medical ICU, First Dept of Internal Medicine, Teaching Hospital, Faculty of Medicine and Biomedical Center in Pilsen, Charles University, Pilsen, Czech Republic. ${ }^{31}$ Dept of Anesthesia, Intensive Care, and Pain Therapy, University of Foggia, Policlinico "00 Riuniti", Foggia, Italy. ${ }^{32}$ Dept of Intensive Care Medicine, St Olav's University Hospital, Trondheim, Norway. ${ }^{33}$ Dept of Anesthesiology, Herlev University Hospital, UCPH, Herlev, Denmark. ${ }^{34}$ Réanimation Polyvalente et Département d'Anesthésie et de Réanimation, Institut Paoli-Calmettes, Marseille, France. ${ }^{35} \mathrm{~A}$ list of collaborators can be found in the Acknowledgements section.

Correspondence: Philippe R. Bauer, Mayo Clinic, Division of Pulmonary and Critical Care Medicine, Gonda 18, 200 First Street SW, Rochester, MN 55905, USA. E-mail: Bauer.Philippedmayo.edu 
bronchoscopy and mortality, and to assess the complications associated with bronchoscopy including respiratory deterioration or unanticipated cardiac arrest. Our hypothesis was that bronchoscopy, with limited complications, would reduce the number of unidentified causes of respiratory failure and be associated with reduced hospital mortality.

\section{Methods}

Study methodology has been described elsewhere [13]. In brief, 1611 patients were enrolled in 62 intensive care units (ICUs) from 16 countries between October 2015 and June 2016 with a 3-month follow-up. We included adult patients with known immunosuppression (immunosuppressive drugs, solid tumour, solid organ transplant, haematological malignancies and haematological stem cell transplant) who were admitted to the ICU with acute hypoxaemic respiratory failure (respiratory rate $>30$ breaths $\mathrm{min}^{-1}$, laboured breathing, arterial oxygen saturation measured by pulse oximetry $<90 \%$ or arterial oxygen tension $\left(\mathrm{PaO}_{2}\right)<60 \mathrm{mmHg}$ on room air or need for $>4 \mathrm{~L} \cdot \mathrm{min}^{-1}$ oxygen, or need for mechanical ventilation). Patients were excluded if they were admitted to the ICU after cardiac arrest, or only to secure bronchoscopy, had elective surgery within 6 days, or had respiratory failure occurring $<7$ days after solid organ transplant.

The primary outcome was in-hospital mortality. The primary exposure was bronchoscopy performed in the ICU. Covariates included demographics, type of immunosuppression, comorbidities, Sequential Organ Failure Assessment (SOFA) score, symptoms and degree of respiratory failure, all measured at baseline. Two groups, those who underwent bronchoscopy in the ICU coupled with noninvasive testing and those who did not get bronchoscopy but only noninvasive testing, were compared with each other by bivariate analyses. Results are expressed as median and interquartile range or number and percentage as appropriate. Wilcoxon rank sum test or Fisher's exact test was used to compare variables between groups as appropriate. Potential confounders were defined as any available variable on admission that could be associated with bronchoscopy, had a prognostic value, and did not reside in the causal pathway between bronchoscopy and hospital mortality [14]. To adjust for any of those variables, logistic regression and propensity matching were used [15]. We developed a propensity score logistic model based on the probability of having received bronchoscopy and then matched individuals on the basis of their propensity score using a 1:1 matching algorithm without replacement within a calliper of $0.1 \mathrm{SD}$ of the logit of the propensity score. To handle missing data in confounders, MICE (multiple imputation with chained equations) was used for the model, where the propensity score for each patient was averaged across 30 completed datasets (including those confounders and some auxiliary variables such as sex and tobacco use, as well as the outcomes as recommended), while matching used these averaged scores to estimate the treatment effect [16]. Imbalances, before and after matching, were measured by standardised mean differences and area under the curve of the propensity score model [17]. To test for centre effect, we plotted outcome by centre and subsequently used a funnel plot to test for heterogeneity. Several sensitivity analyses were also performed. All analyses were performed using R version 3.3.2 (www.R-project.org); all tests were two-sided with p-values $\leqslant 0.05$ denoting statistical significance.

\section{Results}

\section{Patient characteristics}

Of the 1611 patients, 24 lacked information on bronchoscopy and were excluded (figure 1). Of the remaining 1587 patients, 618 (39\%) underwent flexible fibreoptic bronchoscopy coupled with noninvasive investigations and 969 (61\%) underwent noninvasive investigations alone (table 1).

Patients in the bronchoscopy group were younger, had a longer duration of symptoms and were admitted later to the ICU after hospital admission than those in the nonbronchoscopy group. More patients in the bronchoscopy group had haematological disease, haematopoietic stem cell transplant and solid organ transplant. They were less likely to have a solid organ tumour or chronic obstructive pulmonary disease (COPD), and more likely to have a higher Eastern Cooperative Oncology Group performance status score (supplementary tables S1 and S2). SOFA score on admission was higher, notably for its respiratory and haematological components (supplementary table S3). On admission, patients in the bronchoscopy group had more respiratory symptoms, higher arterial partial pressure of carbon dioxide and lower $P_{\mathrm{aO}_{2}} /$ inspiratory oxygen fraction $\left(\mathrm{FIO}_{2}\right)$ ratio (supplementary table S4). Initial goals of care were different, with more "full-code" directives on admission in the bronchoscopy group.

\section{Diagnosis}

In the bronchoscopy group, the cause of acute respiratory failure was less often identified on admission, in spite of having more pre-ICU bronchoscopies. Although noninvasive testing was performed more often, the rate of unidentified cause by noninvasive testing alone remained higher (table 2). Specifically, there were fewer positive blood cultures and fewer positive urine antigens (e.g. Streptococcus pneumoniae); 
FIGURE 1 Study flowchart with initial matching $(n=526)$ and subsequent matching $(n=494)$ adding the variables "bronchoscopy prior to intensive care unit admission", "goal of care discussion" and "disease stage status".

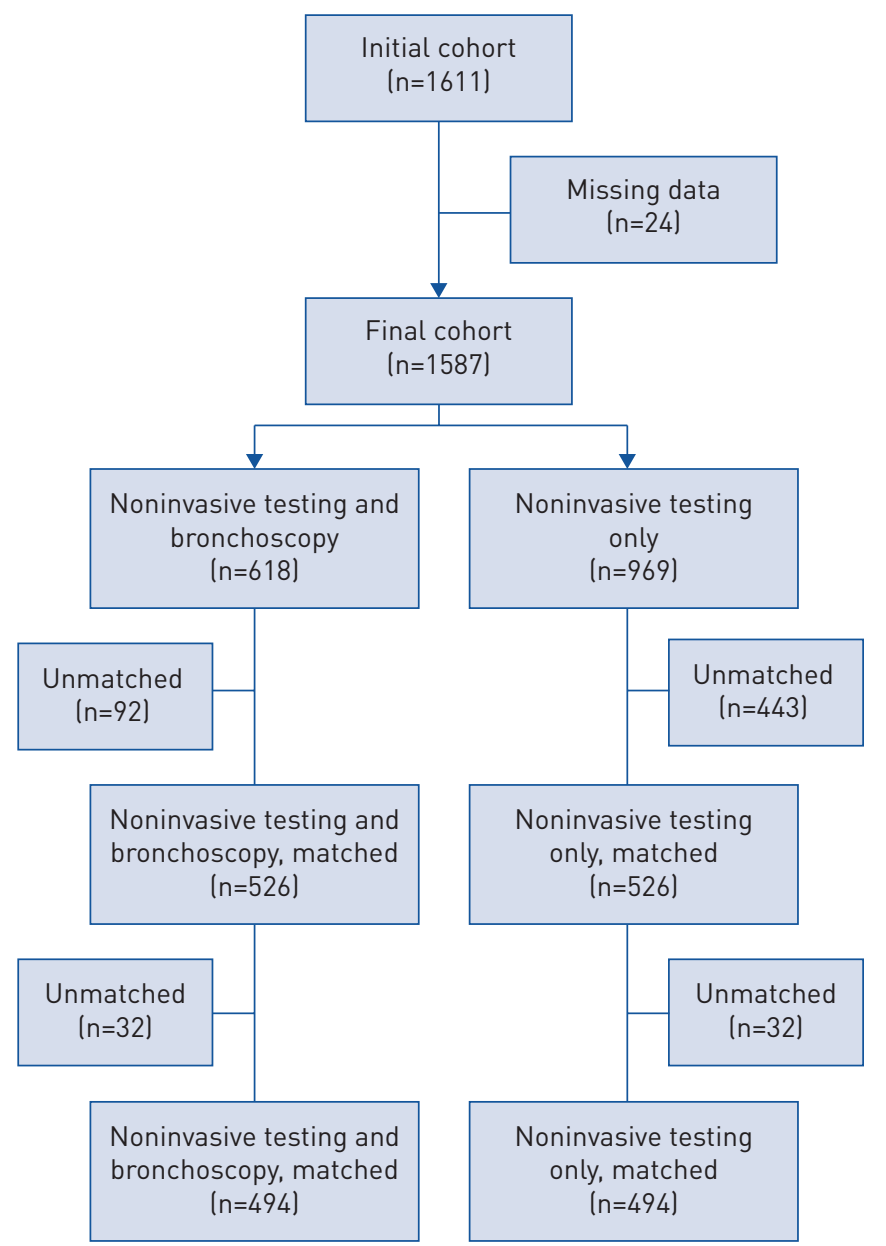

C-reactive protein values were higher. On chest radiography and computed tomography scan, alveolar opacities involved more quadrants, with more evidence of fibrosis (supplementary table S5). After bronchoscopy, the rate of unidentified cause reached $13 \%$ and was identical to the nonbronchoscopy group. Open lung biopsy was rarely performed: $13(2 \%)$ patients in the bronchoscopy group and six (1\%) patients in the nonbronchoscopy group. There were more infectious causes in the bronchoscopy group, including more diagnoses for $P$. jirovecii (supplementary table S6).

Among the 618 patients who underwent bronchoscopy in the ICU (supplementary table S7), at least 90\% underwent bronchoalveolar lavage and a few had either protected bacterial distal sampling (14\%) or transbronchial biopsy (5\%). Bronchoscopy was most commonly performed by the intensivist (55\%) and less often by an external consultant (34\%). It was the first-line diagnostic strategy in $45 \%$. The majority of patients who had bronchoscopy were intubated and ventilated (61\%), 13\% were receiving standard oxygen, $4 \%$ high-flow nasal cannula therapy and $4 \%$ noninvasive ventilation; the type of respiratory support was not specified in $18 \%$ of patients. Respiratory status worsened after bronchoscopy in $11 \%$ of patients; clinical details of worsening respiratory status were not collected. Diagnosis was obtained by bronchoscopy in $49 \%$ of patients and achieved by noninvasive testing methods in $22 \%$ as well, giving an adjusted diagnosis yield of $27 \%$. The therapeutic yield was $38 \%$ and prompted a change in management, adding a new treatment in $12 \%$ or withdrawing antibiotics in $26 \%$. There was no difference in the diagnosis achieved by bronchoscopy between intensivist and external consultant, but the therapeutic yield was greater when the bronchoscopy was performed by an intensivist ( $48 \%$ versus $35 \%$; $\mathrm{p}=0.01$ ).

\section{Outcome}

Overall, more patients in the bronchoscopy group were intubated and were also intubated sooner than in the nonbronchoscopy group (table 3). The bronchoscopy group presented with more cases of septic shock and required more ICU support (e.g. fluids, vasopressors and renal replacement therapy). The bronchoscopy group was also more often on immunosuppressive medications, including corticosteroids, within the first 7 days of ICU admission. The perception of complexity of medical and ICU management 
TABLE 1 Characteristics of patients according to diagnostic group

\begin{tabular}{|c|c|c|c|c|c|}
\hline & \multicolumn{2}{|r|}{ NIT-FOB } & \multicolumn{2}{|r|}{ NIT } & \multirow[t]{2}{*}{ p-value } \\
\hline & $\mathbf{n}$ & Statistics & $\mathbf{n}$ & Statistics & \\
\hline Subjects & 618 & & 969 & & \\
\hline \multicolumn{6}{|l|}{ Sex } \\
\hline Male & 356 & 58.3 & 583 & 60.4 & 0.43 \\
\hline Female & 255 & 41.7 & 382 & 39.6 & \\
\hline Age years & 587 & $62.3(53.3-69.9)$ & 927 & $64.7(55.2-72.4)$ & 0.0009 \\
\hline Height $\mathrm{cm}$ & 588 & $170(162-175)$ & 889 & $170(163-177)$ & 0.073 \\
\hline Weight kg & 610 & $73(62-84)$ & 922 & $72(62-85)$ & 0.63 \\
\hline Duration of symptoms days & 586 & $2(0-6)$ & 936 & $1(0-3)$ & $<0.0001$ \\
\hline \multicolumn{6}{|l|}{ Location before ICU } \\
\hline Emergency room & 93 & 16.3 & 251 & 27.5 & $<0.0001$ \\
\hline Ward & 347 & 61.0 & 505 & 55.3 & \\
\hline Other & 129 & 22.7 & 157 & 17.2 & \\
\hline Same day ICU admission & 160 & 26.4 & 355 & 37.0 & $<0.0001$ \\
\hline SOFA score on admission & 615 & $8(4-10.5)$ & 960 & $7(4-10)$ & 0.0009 \\
\hline Duration of disease days & 344 & $166(21-593)$ & 595 & $113(11-728)$ & 0.34 \\
\hline Haematological malignancy any & 346 & 56.0 & 482 & 49.7 & 0.017 \\
\hline \multicolumn{6}{|l|}{ Haematopoietic stem cell transplant } \\
\hline Autologous & 44 & 7.1 & 56 & 5.8 & $<0.0001$ \\
\hline Allogeneic & 82 & 13.3 & 69 & 7.1 & \\
\hline Systemic disease & 124 & 20.1 & 153 & 15.8 & 0.034 \\
\hline Solid organ tumour & 168 & 27.2 & 382 & 39.4 & $<0.0001$ \\
\hline Solid organ transplant & 78 & 14.1 & 63 & 7.3 & $<0.0001$ \\
\hline Corticosteroid use & 495 & $0(0-22)$ & 802 & $0(0-15)$ & 0.006 \\
\hline Neutropenia & 95 & 16.4 & 154 & 16.4 & 1.00 \\
\hline
\end{tabular}

Data are presented as \% or median (interquartile range), unless otherwise stated. NIT-FOB: noninvasive testing coupled with fibreoptic bronchoscopy; NIT: noninvasive testing only; ICU: intensive care unit; SOFA: Sequential Organ Failure Assessment.

was higher. Invasive fungal infections, drug toxicity and ventilator-associated pneumonias were more frequent. Cardiac arrest after intubation was rare: 20 (1\%) patients and the frequency was the same in both groups. During ICU stay, end-of-life discussions led to more conversion to do-not-resuscitate status in the bronchoscopy group. Duration of mechanical ventilation and ICU length of stay were longer, and ICU, hospital and 90-day mortality were higher, in the bronchoscopy group (table 3). There was no difference in hospital mortality when the bronchoscopy was performed by an external consultant, with 39\% ICU mortality and $47 \%$ hospital mortality versus $42 \%$ and $49 \%$, respectively, when performed by an intensivist. Among 167 (27\%) patients for whom bronchoscopy was both first-line diagnostic strategy and achieved diagnosis alone, $74(31 \%)$ died in the ICU and 85 (36\%) died in the hospital, which is in agreement with estimates from the whole cohort (32\% and $42 \%$, respectively). Of the $236(38 \%)$ patients with treatment

TABLE 2 Causes and diagnosis yield of acute respiratory failure according to diagnostic group

\begin{tabular}{lcccc} 
& Total & NIT-FOB & NIT & p-value \\
\hline Subjects & 1587 & 618 & 969 & \\
Infectious & $938(59)$ & $421(68)$ & $517(53)$ & $<0.0001$ \\
$\begin{array}{l}\text { Pneumocystis jirovecii } \\
\text { Noninfectious }\end{array}$ & $69(4)$ & $48(8)$ & $21(2)$ & $<0.0001$ \\
$\begin{array}{l}\text { Diagnosis } \\
\quad \text { Identified on admission }\end{array}$ & $440(28)$ & $112(18)$ & $328(34)$ & $<0.0001$ \\
$\quad$ Identified with noninvasive testing & $825(52)$ & $263(43)$ & $562(58)$ & $<0.0001$ \\
$\quad$ Identified with bronchoscopy alone & $318(20)$ & $135(22)$ & $283(29)$ & 0.0125 \\
$\quad 167(11)$ & $167(27)$ & & \\
$\quad$ Unidentified & $209(13)$ & $85(14)$ & $124(13)$ & 0.595 \\
\hline
\end{tabular}

Data are presented as $\mathrm{n}$ or $\mathrm{n}(\%)$, unless otherwise stated. NIT-FOB: noninvasive testing coupled with fibreoptic bronchoscopy; NIT: noninvasive testing only. " : excluding those with unknown diagnosis. 
TABLE 3 Outcome according to diagnostic group

\begin{tabular}{|c|c|c|c|c|c|}
\hline & \multicolumn{2}{|r|}{ NIT-FOB } & \multicolumn{2}{|r|}{ NIT } & \multirow[t]{2}{*}{ p-value } \\
\hline & $\mathrm{n}$ & Statistics & $\mathbf{n}$ & Statistics & \\
\hline Subjects & 618 & & 969 & & \\
\hline Intubation & 533 & 86.3 & 446 & 46.0 & $<0.0001$ \\
\hline \multicolumn{6}{|l|}{ Timing of intubation } \\
\hline $0-48 \mathrm{~h}$ & 410 & 66.3 & 370 & 38.2 & $<0.0001$ \\
\hline$>48 \mathrm{~h}$ & 123 & 19.9 & 76 & 7.8 & \\
\hline \multicolumn{6}{|l|}{ Goal of care on admission } \\
\hline Full code & 522 & 84.5 & 723 & 74.6 & $<0.0001$ \\
\hline ICU trial & 20 & 3.2 & 49 & 5.1 & \\
\hline Early ICU & 11 & 1.8 & 17 & 1.8 & \\
\hline $\mathrm{DNI}$ & 3 & 0.5 & 55 & 5.7 & \\
\hline DNR & 10 & 1.6 & 44 & 4.5 & \\
\hline None/unknown & 52 & 8.4 & 81 & 8.4 & \\
\hline Fluids expansion on day $1 \mathrm{~mL}$ & 554 & $1500(500-3000)$ & 873 & $1170(300-2500)$ & 0.0007 \\
\hline Vasopressors days 1-7 & 455 & 73.6 & 467 & 48.2 & $<0.0001$ \\
\hline Low-dose steroids days 1-7 & 221 & 39.0 & 287 & 33.0 & 0.023 \\
\hline High-dose steroids days $1-7$ & 162 & 28.2 & 169 & 19.0 & $<0.0001$ \\
\hline Septic shock days $1-7$ & 329 & 53.2 & 349 & 36.0 & $<0.0001$ \\
\hline Dialysis days 1-7 & 142 & 23.0 & 153 & 15.8 & 0.0004 \\
\hline \multicolumn{6}{|l|}{ End-of-life decision } \\
\hline None & 421 & 68.1 & 731 & 75.4 & 0.0004 \\
\hline No escalation & 60 & 9.7 & 79 & 8.2 & \\
\hline Withholding & 33 & 5.3 & 62 & 6.4 & \\
\hline Withdrawing & 104 & 16.8 & 97 & 10.0 & \\
\hline ICU length of stay days & 598 & $11(6-19)$ & 955 & $5(2-9)$ & $<0.0001$ \\
\hline Hospital length of stay days & 511 & $28(16-49)$ & 835 & $18(9-34)$ & $<0.0001$ \\
\hline ICU mortality & 248 & 40.1 & 267 & 27.6 & $<0.0001$ \\
\hline Hospital mortality & 294 & 49 & 378 & 41 & 0.003 \\
\hline 90-day mortality & 325 & 60.5 & 436 & 53.7 & 0.016 \\
\hline
\end{tabular}

Data are presented as \% or median (interquartile range), unless otherwise stated. NIT-FOB: noninvasive testing coupled with fibreoptic bronchoscopy; NIT: noninvasive testing only; ICU: intensive care unit; DNI: do not intubate; DNR: do not resuscitate.

change only due to bronchoscopy, 88 (37\%) died in the ICU and 103 (44\%) died in the hospital, which is also close to that observed in the whole cohort.

All identifiable confounders (supplementary table S8) were imbalanced between both groups, as measured by their standardised mean difference (supplementary table S9 and supplementary figure S1), and thus were included in the model [18]. Of the 618 patients in the bronchoscopy group, 92 could not be matched, leaving a matching sample size of 526 in each group (supplementary figure S2). Before matching, performing a bronchoscopy in the ICU was associated with increased odds of death of $38 \%$ and $41 \%$ after matching for age, performance status, symptoms, severity, type of immunosuppression, type of admission and respiratory status (table 4 and figure 2). Although we found some heterogeneity in outcome among centres, there was no evidence of centre effect for diagnostic testing (supplementary figure S3). We also found no change in the result when the variable "bronchoscopy before ICU admission" was added to the model. Although hospital mortality ranged from $32 \%$ in patients whose disease was in remission up to $56 \%$ in those whose disease was uncontrolled, the odds of dying did not change, and remained $41 \%$ higher in the bronchoscopy group when goals of care and disease severity were also added to the model. Hospital mortality was $49 \%$ in the bronchoscopy group overall and $44 \%$ (55 out of 124) in patients with bronchoscopy who could not be matched and were not included in the propensity score logistic model.

\section{Discussion}

In this study of acute hypoxaemic respiratory failure in immunocompromised patients around the world, bronchoscopy was performed in less than half of patients and yielded a diagnosis in about half of the cases. Bronchoscopy influenced management in approximately one-third of patients. It was performed more often in intubated patients and was associated with some risk of respiratory deterioration. With bronchoscopy, the rate of undiagnosed causes, which was higher in that group otherwise, became, after 
TABLE 4 Estimate of the effect of bronchoscopy on hospital mortality

\begin{tabular}{lcccc} 
Model & Patients n & AUC & OR (95\% CI) & p-value \\
\hline Original sample & 618 & 0.726 & $1.38(1.12-1.70)$ & 0.002 \\
Original sample, with random centre effect & 618 & 0.698 & $1.51(1.19-1.91)$ & 0.0005 \\
Matched sample, initial & 526 & 0.4994 & $1.41(1.08-1.81)$ & 0.006 \\
Matched sample, with random centre effect & 526 & 0.699 & $1.11(1.03-1.18)$ & 0.0045 \\
Matched also with “bronchoscopy prior to ICU" feature & 526 & 0.5013 & $1.41(1.09-1.84)$ & 0.010 \\
Matched also with "goals of care" and "disease status" & 494 & 0.4980 & $1.41(1.08-1.81)$ & 0.006 \\
\hline
\end{tabular}

AUC: area under the curve; ICU: intensive care unit.

bronchoscopy, identical to that in the nonbronchoscopy group. Bronchoscopy was associated with increased ICU and hospital mortality, even after adjusting for multiple severity criteria. Several reasons for this association are possible and could include: 1) bronchoscopy itself may increase the risk of mortality in this population, 2) bronchoscopy may be just a marker of disease severity, 3) bronchoscopy may be just a marker of underlying diagnoses with worse prognoses or without effective therapies, as eventually bronchoscopy was performed out of "desperation", or 4) one or more unmeasured confounders associated with ICU bronchoscopy are driving the increased risk of mortality.

What are some limitations of this study? First, data collection methods were predetermined [19] and therefore subject to data selection bias [20]. Second, the use of bronchoscopy was heterogeneous and may have reflected a different case mix or different practices. We did not collect data on the timing of bronchoscopy relative to ICU admission, the volume of fluid used for the lavage or the timing and severity of respiratory worsening following bronchoscopy. Third, ICU severity was assessed by SOFA score and not Acute Physiologic Assessment and Chronic Health Evaluation (APACHE), although SOFA has proven to be superior to APACHE II to predict hospital mortality in allogeneic stem cell transplant recipients [21]. Fourth, we used a propensity score approach that explicitly modelled the treatment selection assignment process using the observed background characteristics. However, this assumed that there were no unmeasured confounders. Otherwise, to remove the bias caused by unmeasured confounding, instrumental variable analysis would be an alternative approach provided that key assumptions (some of which are also untestable) were met. Notably, the effect of the instrumental variable on the dependent measure should be entirely mediated via its effect on exposure assignment. In ICU settings, previously used instrumental variables consisted of the bed availability at the time of assessment for ICU transfer [22, 23] or the physician's main specialisation [24]. Unfortunately, such instruments were not measured in our study. The need for reporting such variables in ICU cohorts should be highlighted for further studies.
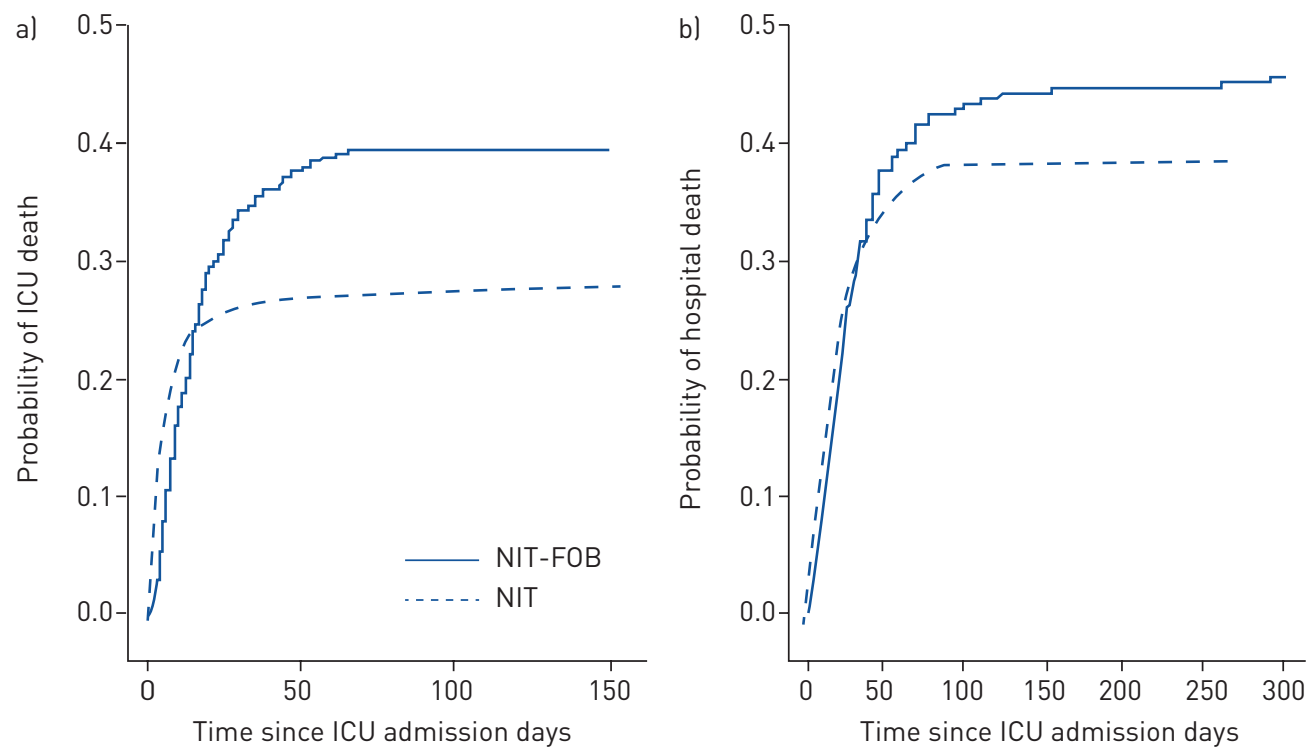

FIGURE 2 Observed cumulative risk of death in the a) intensive care unit (ICU) or b) hospital after matching on propensity score. NIT-FOB: noninvasive testing coupled with fibreoptic bronchoscopy; NIT: noninvasive testing only. 
Is bronchoscopy harmful? In a prospective observational study of 148 patients with malignancy, $49 \%$ of nonintubated patients had respiratory deterioration and $36 \%$ required ventilator support [5]. In a subsequent randomised trial of 219 patients [8], the need for mechanical ventilation was no different with (35\%) or without $(39 \%)$ bronchoscopy and the proportion of patients that remained without diagnosis was the same with $(22 \%)$ or without $(20 \%)$ bronchoscopy. In both studies, hospital mortality did not differ between the two groups [5, 8]. Risk factors of death were not linked to bronchoscopy, but to disease characteristics, failure to identify the cause of acute respiratory failure and the need for mechanical ventilation or vasopressors [5]. In another study in 169 patients with acute respiratory failure, bronchoscopy was complicated by an increase in respiratory support within the subsequent $24 \mathrm{~h}$ in $35 \%$ of cases, of which $15 \%$ led to intubation; COPD and immunosuppression were associated with need for invasive mechanical ventilation [25]. In an observational study of 164 intubated patients, bronchoscopy and bronchoalveolar lavage were frequently associated with a decline in the $\mathrm{PaO}_{2} / \mathrm{FIO}_{2}$ ratio (29\%) within $1 \mathrm{~h}$ and haemodynamic instability (22\%) within $24 \mathrm{~h}$ following the procedure [26].

Our rate of $13 \%$ unidentified cause is identical to recent data in the literature showing that failure to identify a cause is associated with a higher rate of invasive mechanical ventilation and hospital mortality [10]. In our study, bronchoscopy corrected the diagnostic gap with the noninvasive group but did not reduce further the rate of unidentified cause. Bronchoscopy was also complicated by worsening respiratory failure in some patients. Although the timing of bronchoscopy relative to intubation was not collected, more patients in this group were intubated and mechanically ventilated sooner after arrival in the ICU than in the nonbronchoscopy group and we suspect it was either for the purpose of the procedure or as a consequence, or because of their degree of respiratory failure, possible delayed ICU admissions or other conditions such as shock [2, 27]. Despite having balanced the propensity score with identifiable confounders, we may have unmasked confounders such as intubation itself which influences both bronchoscopy and outcome [28]. Although the patients in the bronchoscopy group were more severely ill, the effect of bronchoscopy persisted after adjustment for severity. Clinicians tend to underestimate harms and overestimate benefits of testing [29], and we suspect that bronchoscopy itself could be a marker of severity, i.e. a case of over-testing in a desperate attempt to find a diagnosis.

Can bronchoscopy be beneficial? Our findings are very similar to a small retrospective study of 35 patients with neutropenic fever and pulmonary infiltrates in which bronchoscopy yielded a diagnosis in 49\% [11]. Consequently, a change in management occurred in $51 \%$ of patients. Although complications were limited (9\%), the 28-day mortality was highest in patients who were mechanically ventilated prior to bronchoscopy. Similar findings were obtained in a small retrospective study of 26 bronchoscopies with a positive sampling in $23 \%$ of patients, a change in management in $38 \%$ and a low complication rate (8\%) [30]. The same was also observed in a study comparing bronchoalveolar lavage alone or combined with protected specimen brushing, yielding a diagnosis in $43 \%$ and $57 \%$, respectively [31]. Combining noninvasive testing with bronchoscopy more often yielded a diagnosis (70\%) and altered management $(55 \%)$ in a prospective series of 128 patients with neutropenic fever and pulmonary infiltrates, and survival was higher when the diagnosis was made early [32]. A randomised trial comparing noninvasive testing alone or coupled with bronchoscopy in nonintubated patients demonstrated that information added with bronchoscopy was relatively modest $(18 \%)$, but the procedure was safe with no impact on the rate of intubation or 28-day survival rate [8]. A French expert panel recommended that the search for a diagnosis should be a primary objective in acute respiratory failure in immunocompromised and neutropenic patients, with early management being associated with a better risk/benefit ratio. It should systematically consider type of malignancy, immunosuppression, prophylaxis and clinical presentation of the failure, in order to establish a pre-test probability that will better define the respective role of noninvasive and invasive procedures such as bronchoscopy [33]. The British Thoracic Society guidelines recommended that bronchoscopy with bronchoalveolar lavage be considered in immunosuppressed patients to provide diagnostic information (Grade C) when a diagnosis is not likely to be obtained noninvasively [34]. Bronchoscopy is instrumental in identifying specific infectious causes, such as Aspergillus and P. jirovecii [35], or noninfectious causes, such as diffuse alveolar haemorrhage. Delaying diagnosis of pneumocystis pneumonia is associated with worse outcome [36]. Galactomannan testing in bronchoalveolar lavage is highly specific and more sensitive than serum testing [37]. Finding an infectious aetiology with early change in antibiotic treatment is associated with better outcomes [38]. However, in the era of empiric therapy with broad-spectrum antibiotics and antifungal agents, a recent retrospective study of 101 patients, including children, with cancers or haematopoietic stem cell transplant showed that bronchoscopy led to few changes in management $(33 \%)$ and few complications except when biopsies were performed [39]. In 71 patients with haematological malignancies, bronchoscopy, indicated for respiratory failure or sepsis, led to a change in management in $45 \%$ of patients while complications occurred in $13 \%$, some serious and leading to prolonged mechanical ventilation, need for tracheostomy and increased risk of death. Age, sex, APACHE II score and active chemotherapy did not predict management change or complications 
post-bronchoscopy [40]. In our study, indiscriminate bronchoscopy itself may have contributed to worse outcome. The finding of this large prospective, multicentre, multinational observational study is likely representative and generalisable. Patients were severely ill, had a high intubation rate and high mortality. This study suggests that the use of bronchoscopy should be individualised and tailored to specific needs (e.g. nonbacterial infectious cause) in immunosuppressed patients.

\section{Conclusion}

In this large ICU cohort of immunosuppressed patients with acute hypoxaemic respiratory failure, bronchoscopy was associated with improved diagnosis and changes in management, but increased ICU and hospital mortality, even after adjusting for severity and other identifiable confounders. Rethinking bronchoscopy in terms of pre- and post-test probability and balancing risk and benefit in individualised cases should be investigated further.

Acknowledgements: This study was performed on behalf of the CarIng for CrItIcally Ill Immuno-compromIsed PatIents MultInatIonal Network (Nine-I). This group includes critical care specialists from 16 countries in Europe, the USA, Canada and South America. The primary aim of this group is to improve and standardise practices in the management of critically ill immunocompromised patients.

Collaborators: Antoine Rabbat (Hôpital Cochin, Paris, France); Michael Darmon (CHU, Saint-Etienne, France); Kada Klouche (CHU, Montpellier, France); Laura Platon (CHU, Montpellier, France); Julien Mayaux (CHU Pitié-Salpétrière, Paris, France); Akli Chermak (CH Sud Essonne, Etampes, France); Caroline Lemaitre (University Hospital, Rouen, France); Elise Artaud-Macari (University Hospital, Rouen, France); Jonas Nelsen (Rigshospitalet, Copenhagen, Denmark); Thomas Kaufmann (University Medical Center Groningen, Groningen, The Netherlands); William Viana (Hospital Copa d'Or, Rio de Janeiro, Brazil); Thiago Lishoa (Hospital Santa Rita, Santa Casa de Misericordia, Porte Allegre, Brazil); Thiago Domingos Correa (Hospital Israelita Albert Einstein, São Paulo, Brazil); Belen Encina (Val d'Hebron Institut of Research, Barcelona, Spain); Antonio Socias (Hospital Son Llatcer, Palma de Mallorca, Spain); Rafael Mañez (Hospital de Bellvitge, Barcelona, Spain); Emilio Rodriguez-Ruis (Hospital Clinico Universitario, Santiago de Compostela, Spain); Dominique Benoit (Ghent University Hospital, Ghent, Belgium); Thomas Staudinger (Medical University of Vienna, Vienna, Austria); Gottfried Heinz (Medical University of Vienna, Vienna, Austria); Gürkan Sengölge (Medical University of Vienna, Vienna, Austria); Christian Zauner (Medical University of Vienna, Vienna, Austria); Peter Jaksch (Medical University of Vienna, Vienna, Austria); Aisling McMahon (St James's Hospital, Dublin, Ireland); Balik Martin (General University Hospital, Prague, Czech Republic); Gilda Cinnella (University of Foggia, Foggia, Italy); Sumech Shah (Mount Sinai Hospital, Toronto, ON, Canada); Pleun Hemelaar (Radboud University Medical Center, Nijmegen, The Netherlands); Fabio Silvio Taccone (Hôpital Erasme, Université Libre de Bruxelles, Brussels, Belgium); Jorge Salluh (Hospital Copa D’Or, Rio de Janeiro, Brazil); Peter Schellongowski (Medical University of Vienna, Vienna, Austria); Katerina Rusinova (Charles University and General University Hospital, Prague, Czech Republic); Nicolas Terzi (CHU Grenoble-Alpes, Grenoble, France); Achille Kouatchet (University Hospital of Angers, Angers, France); Miia Valkonen (University of Helsinki and Helsinki University Hospital, Helsinki, Finland); Fabrice Bruneel (Center Hospitalier de Versailles, Le Chesnay, France); Frédéric Pène (Cochin Hospital and University Paris Descartes, Paris, France); Anne Sophie Moreau (CHU Lille and University of Lille, Lille, France); Christophe Girault (Normandie University and Rouen University Hospital, Rouen, France); Ulysses V.A. Silva (Fundação Pio XII, Hospital de Câncer de Barretos, Barretos, Brazil); Luca Montini (IRCCS Fondazione Policlinico Universitario Agostino Gemelli, Università Cattolica del Sacro Cuore, Rome, Italy); François Barbier (La Source Hospital, CHR Orléans, Orléans, France); Benjamin Gaborit (Hôtel Dieu-HME, University Hospital of Nantes, Nantes, France); William N. Viana (Hospital Copa D’Or, Rio de Janeiro, Brazil); Ana Paula P. de Moraes (Hospital de Câncer do Maranhao, São Luis, Brazil); Guilliana M. Moralez (Hospital Getulio Vargas, Rio de Janeiro, Brazil); Isabelle Vinatier (CHD de Vendée, La Roche Sur Yon, France).

Conflict of interest: P.R. Bauer has nothing to disclose. S. Chevret has nothing to disclose. H. Yadav has nothing to disclose. S. Mehta has nothing to disclose. P. Pickkers has nothing to disclose. R.B. Bukan has nothing to disclose. J. Rello has nothing to disclose. A. van de Louw has nothing to disclose. K. Klouche has nothing to disclose. A-P. Meert has nothing to disclose. I. Martin-Loeches has nothing to disclose. B. Marsh has nothing to disclose. L. Socias Crespi has nothing to disclose. G. Moreno-Gonzalez has nothing to disclose. N. Buchtele has nothing to disclose. K. Amrein reports grants and personal fees for lecturing from Fresenius Kabi, personal fees for lecturing and advisory board work from Vifor Pharma and Shire, outside the submitted work. M. Balik has nothing to disclose. M. Antonelli has nothing to disclose. M. Nyunga has nothing to disclose. A. Barratt-Due has nothing to disclose. D.C.J.J. Bergmans has nothing to disclose. A.M.E. Spoelstra-de Man has nothing to disclose. A. Kuitunen has nothing to disclose. F. Wallet has nothing to disclose. A. Seguin has nothing to disclose. V. Metaxa has nothing to disclose. V. Lemiale has nothing to disclose. G. Burghi has nothing to disclose. A. Demoule reports grants, personal fees and nonfinancial support from Philips, personal fees for lecturing and advisory board work from Baxter, personal fees for lecturing from Hamilton, grants and nonfinancial support from Fisher \& Paykel, grants from French Ministry of Health, outside the submitted work. T. Karvunidis has nothing to disclose. A. Cotoia has nothing to disclose. P. Klepstad has nothing to disclose. A.M. Møller has nothing to disclose. D. Mokart has nothing to disclose. E. Azoulay reports personal fees for lecturing and travel support for conference attendance from Gilead, personal fees for lecturing from Baxter, personal fees for lecturing and nonfinancial support from Alexion, grants from Ablynx and MSD, and devices for trials from Fisher \& Paykel, outside the submitted work.

Support statement: This work was supported by the Groupe de Recherche en Réanimation Respiratoire en Onco-Hématologique (Grrr-OH). Funding information for this article has been deposited with the Crossref Funder Registry. 


\section{References}

1 Azoulay E, Soares M, Darmon M, et al. Intensive care of the cancer patient: recent achievements and remaining challenges. Ann Intensive Care 2011; $1: 5$.

2 Azoulay E, Mokart D, Pène F, et al. Outcomes of critically ill patients with hematologic malignancies: prospective multicenter data from France and Belgium - a Groupe de Recherche en Réanimation Respiratoire en Onco-Hématologique study. J Clin Oncol 2013; 31: 2810-2818.

3 Yadav $\mathrm{H}$, Nolan ME, Bohman JK, et al. Epidemiology of acute respiratory distress syndrome following hematopoietic stem cell transplantation. Crit Care Med 2016; 44: 1082-1090.

4 Seong GM, Lee Y, Hong SB, et al. Prognosis of acute respiratory distress syndrome in patients with hematological malignancies. J Intensive Care Med 2018; in press [https://doi.org/10.1177/0885066617753566].

5 Azoulay E, Mokart D, Rabbat A, et al. Diagnostic bronchoscopy in hematology and oncology patients with acute respiratory failure: prospective multicenter data. Crit Care Med 2008; 36: 100-107.

6 Afessa B, Abdulai RM, Kremers WK, et al. Risk factors and outcome of pulmonary complications after autologous hematopoietic stem cell transplant. Chest 2012; 141: 442-450.

7 Azoulay E, Lemiale V, Mokart D, et al. Acute respiratory distress syndrome in patients with malignancies. Intensive Care Med 2014; 40: 1106-1114.

8 Azoulay E, Mokart D, Lambert J, et al. Diagnostic strategy for hematology and oncology patients with acute respiratory failure: randomized controlled trial. Am J Respir Crit Care Med 2010; 182: 1038-1046.

9 Lachant DJ, Croft DP, McGrane Minton H, et al. Nasopharyngeal viral PCR in immunosuppressed patients and its association with virus detection in bronchoalveolar lavage by PCR. Respirology 2017; 22: 1205-1211.

10 Contejean A, Lemiale V, Resche-Rigon M, et al. Increased mortality in hematological malignancy patients with acute respiratory failure from undetermined etiology: a Groupe de Recherche en Réanimation Respiratoire en Onco-Hématologique (Grrr-OH) study. Ann Intensive Care 2016; 6: 102.

11 Peikert T, Rana S, Edell ES. Safety, diagnostic yield, and therapeutic implications of flexible bronchoscopy in patients with febrile neutropenia and pulmonary infiltrates. Mayo Clin Proc 2005; 80: 1414-1420.

12 Shannon VR, Andersson BS, Lei X, et al. Utility of early versus late fiberoptic bronchoscopy in the evaluation of new pulmonary infiltrates following hematopoietic stem cell transplantation. Bone Marrow Transplant 2010; 45: 647-655.

13 Azoulay E, Pickkers P, Soares M, et al. Acute hypoxemic respiratory failure in immunocompromised patients: the Efraim multinational prospective cohort study. Intensive Care Med 2017; 43: 1808-1819.

14 Lederer DJ, Bell SC, Branson RD, et al. Control of confounding and reporting of results in causal inference studies: guidance for authors from editors of respiratory, sleep, and critical care journals. Ann Am Thorac Soc 2019; 16: 22-28.

15 Stuart EA. Matching methods for causal inference: a review and a look forward. Stat Sci 2010; 25: 1-21.

16 Mitra R, Reiter JP. A comparison of two methods of estimating propensity scores after multiple imputation. Stat Methods Med Res 2016; 25: 188-204.

17 Franklin JM, Rassen JA, Ackermann D, et al. Metrics for covariate balance in cohort studies of causal effects. Stat Med 2014; 33: 1685-1699.

18 Austin PC. A tutorial and case study in propensity score analysis: an application to estimating the effect of in-hospital smoking cessation counseling on mortality. Multivariate Behav Res 2011; 46: 119-151.

19 Nørgaard M, Ehrenstein V, Vandenbroucke JP. Confounding in observational studies based on large health care databases: problems and potential solutions - a primer for the clinician. Clin Epidemiol 2017; 9: $185-193$.

20 Leibovici L, Turjeman A, Paul M. The temptation of large numbers. Clin Microbiol Infect 2018; 24: 931-932.

21 Gilli K, Remberger M, Hjelmqvist H, et al. Sequential Organ Failure Assessment predicts the outcome of SCT recipients admitted to intensive care unit. Bone Marrow Transplant 2010; 45: 682-688.

22 Keele L, Harris S, Grieve R. Does transfer to intensive care units reduce mortality? A comparison of an instrumental variables design to risk adjustment. Med Care 2019; in press [https://doi.org/10.1097/MLR. 0000000000001093 .

23 Grieve R, O’Neill S, Basu A, et al. Analysis of benefit of intensive care unit transfer for deteriorating ward patients: a patient-centered approach to clinical evaluation. JAMA Netw Open 2019; 2: e187704.

24 Pirracchio R, Sprung C, Payen D, et al. Benefits of ICU admission in critically ill patients: whether instrumental variable methods or propensity scores should be used. BMC Med Res Methodol 2011; 11: 132.

25 Cracco C, Fartoukh M, Prodanovic H, et al. Safety of performing fiberoptic bronchoscopy in critically ill hypoxemic patients with acute respiratory failure. Intensive Care Med 2013; 39: 45-52.

26 Schnabel RM, van der Velden K, Osinski A, et al. Clinical course and complications following diagnostic bronchoalveolar lavage in critically ill mechanically ventilated patients. BMC Pulm Med 2015; $15: 107$.

27 Allareddy V, Roy A, Rampa S, et al. Outcomes of stem cell transplant patients with acute respiratory failure requiring mechanical ventilation in the United States. Bone Marrow Transplant 2014; 49: 1278-1286.

28 Liu W, Kuramoto SJ, Stuart EA. An introduction to sensitivity analysis for unobserved confounding in nonexperimental prevention research. Prev Sci 2013; 14: 570-580.

29 Hoffmann TC, Del Mar C. Clinicians' expectations of the benefits and harms of treatments, screening, and tests: a systematic review. JAMA Intern Med 2017; 177: 407-419.

30 Seneviratna A, O'Carroll M, Lewis CA, et al. Diagnostic yield of bronchoscopic sampling in febrile neutropenic patients with pulmonary infiltrate and haematological disorders. Intern Med J 2012; 42: 536-541.

31 Boersma WG, Erjavec Z, van der Werf TS, et al. Bronchoscopic diagnosis of pulmonary infiltrates in granulocytopenic patients with hematologic malignancies: BAL versus PSB and PBAL. Respir Med 2007; 101: 317-325.

32 Panda A, McArdle JR. To bronch or not to bronch? A recurring challenge in neutropenic patients with pulmonary infiltrates. Conn Med 2010; 74: 69-77.

33 Schnell D, Azoulay E, Benoit D, et al. Management of neutropenic patients in the intensive care unit (NEWBORNS EXCLUDED) recommendations from an expert panel from the French Intensive Care Society (SRLF) with the French Group for Pediatric Intensive Care Emergencies (GFRUP), the French Society of Anesthesia and Intensive Care (SFAR), the French Society of Hematology (SFH), the French Society for Hospital Hygiene (SF2H), and the French Infectious Diseases Society (SPILF). Ann Intensive Care 2016; 6: 90. 
34 Du Rand IA, Blaikley J, Booton R, et al. British Thoracic Society guideline for diagnostic flexible bronchoscopy in adults: accredited by NICE. Thorax 2013; 68: Suppl. 1, i1-i44.

35 Svensson T, Lundström KL, Höglund M, et al. Utility of bronchoalveolar lavage in diagnosing respiratory tract infections in patients with hematological malignancies: are invasive diagnostics still needed? Ups J Med Sci 2017; 122: $56-60$.

36 Liu Y, Su L, Jiang SJ, et al. Risk factors for mortality from pneumocystis carinii pneumonia (PCP) in non-HIV patients: a meta-analysis. Oncotarget 2017; 8: 59729-59739.

37 Gupta A, Capoor MR, Shende T, et al. Comparative evaluation of galactomannan test with bronchoalveolar lavage and serum for the diagnosis of invasive aspergillosis in patients with hematological malignancies. J Lab Physicians 2017; 9: 234-238.

38 Rañó A, Agustí C, Jimenez P, et al. Pulmonary infiltrates in non-HIV immunocompromised patients: a diagnostic approach using non-invasive and bronchoscopic procedures. Thorax 2001; 56: 379-387.

39 Batra $\mathrm{S}$, Li B, Underhill $\mathrm{N}$, et al. Clinical utility of bronchoalveolar lavage and respiratory tract biopsies in diagnosis and management of suspected invasive respiratory fungal infections in children. Pediatr Blood Cancer 2015; 62: 1579-1586.

40 Deotare U, Merman E, Pincus D, et al. The utility and safety of flexible bronchoscopy in critically ill acute leukemia patients: a retrospective cohort study. Can J Anaesth 2018; 65: 272-279. 